\title{
Efficacy of acupuncture in the prevention and treatment of chemotherapy-induced nausea and vomiting in patients with advanced cancer: a multi-center, single-blind, randomized, sham-controlled clinical research
}

Qi-Wei Li $i^{1,2}$, Ming-Wei Yu ${ }^{1 *}$, Xiao-Min Wang ${ }^{1 *} \mathbb{D}$, Guo-Wang Yang ${ }^{1}$, Huan Wang ${ }^{1}$, Chen-Xi Zhang ${ }^{1}$, Na Xue ${ }^{1}$, Wei-Ru Xu' ${ }^{1}$, Yi Zhang ${ }^{1}$, Pei-Yu Cheng ${ }^{1}$, Lin Yang ${ }^{1}$, Qi Fu and Zhong Yang ${ }^{1}$

\begin{abstract}
Background: Chemotherapy-induced nausea and vomiting (CINV) is a common and distressing side effect. We conducted this clinical trial to compare the effectiveness of true acupuncture vs. sham acupuncture in controlling chemotherapy-induced nausea and vomiting (CINV) among patients with advanced cancer.

Methods: A total of 134 participants were randomly allocated into true acupuncture (TA) $(n=68)$ and sham acupuncture $(S A)(n=66)$ groups. Participants in both groups received acupuncture session twice on the first day of chemotherapy, and once consecutively on the following 4 days. The primary outcome was using the Common Terminology Criteria for Adverse Events (CTCAE) to assess CINV. The secondary outcome measures were the Eastern Cooperative Oncology Group score (ECOG), Simplified Nutritional Appetite Questionnaire (SNAQ), and Hospital Anxiety and Depression scale (HADS).

Results: Compared to the SA group, the TA group didn't show significant improvement in complete response rates of chemotherapy-induced nausea and vomiting (all $P>0.05$ ). However, the TA group could modestly reduce the severity of nausea (from day-3 to day-21, $\mathrm{P}<0.05$ ) or vomiting (from day-4 to day- $21, \mathrm{P}<0.05$ ), which is notably superior to the control group. Besides, TA promoted the nutritional status of patients with a significantly higher score comparing to the $S A$ group on day 14 (21.82 vs.20.12, $P=0.003$ ) and day 21 (22.39 vs. 20.43, $P=0.001$ ). No apparent differences were found in anxiety and depression assessment between these groups. Participants in both groups were well tolerant of acupuncture therapy. There was no adverse event occurs in our study.
\end{abstract}

Conclusion: Acupuncture as an adjunctive approach could alleviate the severity of chemotherapy-induced nausea and vomiting compared to the sham control, even though the effect of acupuncture in preventing CINV occurring is relatively modest.

\footnotetext{
*Correspondence: yumingwei1120@163.com; wangxiaomin_bhtcm@126.

com

${ }^{1}$ Beijing Hospital of Traditional Chinese Medicine Affiliated with Capital Medical University, No 23, Back Road of Art Gallery, Dong Cheng District, Beijing 100010, China

Full list of author information is available at the end of the article
}

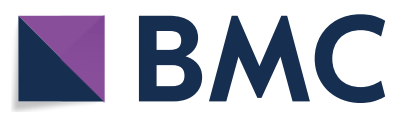

(c) The Author(s) 2020. This article is licensed under a Creative Commons Attribution 4.0 International License, which permits use, sharing, adaptation, distribution and reproduction in any medium or format, as long as you give appropriate credit to the original author(s) and the source, provide a link to the Creative Commons licence, and indicate if changes were made. The images or other third party material in this article are included in the article's Creative Commons licence, unless indicated otherwise in a credit line to the material. If material is not included in the article's Creative Commons licence and your intended use is not permitted by statutory regulation or exceeds the permitted use, you will need to obtain permission directly from the copyright holder. To view a copy of this licence, visit http://creativeco mmons.org/licenses/by/4.0/. The Creative Commons Public Domain Dedication waiver (http://creativecommons.org/publicdomain/ zero/1.0/) applies to the data made available in this article, unless otherwise stated in a credit line to the data. 
Keywords: Acupuncture, Chemotherapy-induced nausea and vomiting, Cancer

\section{Background}

Chemotherapy-induced nausea and vomiting (CINV) is one of the most common and debilitating side effects which exerts a detrimental influence on the quality of life (QOL) of cancer patients and reduces their compliance towards conventional therapies. CINV typically presents in two phases, the acute phase and the delayed phase [1]. Acute CINV occurs within 1-2 h of chemotherapy administration and can last for up to $24 \mathrm{~h}$, while delayed CINV phase presents more than $24 \mathrm{~h}$ after chemotherapy until $120 \mathrm{~h}$.

Nowadays, the development of prophylaxis for chemotherapy-induced emesis has dramatically relieved chemotherapy-induced emesis. Patients receiving highly emetogenic chemotherapy (HEC) are recommended with a four drug combination of a neurokinin $1\left(\mathrm{NK}_{1}\right)$ receptor antagonist, a serotonin $\left(5-\mathrm{HT}_{3}\right)$ receptor antagonist, dexamethasone, and olanzapine by the ASCO guideline [2]. A phase 3 trial reported this combination therapy achieved a complete response rate of $79 \%$ in the patients who were receiving the first circle of cisplatin-based chemotherapy [3]. However, this result was based on only a single circle of chemotherapy with cisplatin. Additionally, patients undergoing chemotherapy with anthracycline and cyclophosphamide had a more inadequate control of CINV in the acute phase than those who treated with cisplatin [4]. It could be speculated that the complete response rate of CINV by the standard antiemetic medications in clinical practice is lower than what is reported in the literature. Despite the advance in antiemetic therapy, practice patterns of antiemetic use revealed low adherence to those guidelines [5] due to unbalanced medical development, financial burdens, provider barriers, and patient factors in different countries and areas $[6,7]$. Although emesis could be prevented well in most cases, nausea mainly occurring in delay-phase remains a significant challenge for cancer patients $[4,8,9]$.

Efforts should be made to explore the economic and practical approaches for better symptom control of CINV. Integrative therapies, including acupressure and electroacupuncture, are recommended for clinical application with fewer side effects and high feasibility by ASCO guideline [10]. Acupuncture, as a component of traditional medicine systems, has been practiced in Asia for thousands of years and been well accepted worldwide. By stimulation on specific acupoints beneath the skin, acupuncture accommodates the flow of energy called $q i$ throughout the body [11] and removes the blockages in meridians helping the body reestablishing homeostasis.
Traditional Chinese acupuncture requires exerting manipulation on needles to present a sensation of $d e q i$ (a soreness, fullness, heaviness, or local area distension [12]).

It is reported by researchers from different countries with the optimized outcomes of acupuncture in controlling chemotherapy-induced emesis [12, 13]. Acupuncture could be considered as a supplementary therapy for relieving symptoms of CINV. Nevertheless, owing to the limitation of research quality, although acupuncture could be an appropriate adjunctive treatment for CINV, it still needs evidence of high-quality clinical researches according to a systematic review [14]. In our study, we performed a multi-center, single-blind, randomized, controlled trial to investigate the efficacy and safety of acupuncture in the prevention and treatment of chemotherapy-induced nausea and vomiting in patients with advanced cancer.

\section{Methods}

This study was a multi-center, single-blind, randomized, sham-controlled clinical trial designed to investigate the effect of acupuncture versus sham acupuncture on CINV symptoms in patients with advanced cancer. All research procedures were approved by the Research Ethical Committee of Beijing Hospital of Traditional Chinese Medicine Affiliated to Capital Medical University (ref: 2014BL-067). All patients included in this study provided written, informed consent. All investigations were conducted in accordance with the Consolidated Standards of Reporting Trials statement [15] and the 2010 Standards for Reporting Interventions in Clinical Trials of Acupuncture [16]. The study protocol was registered on ISRCTN and Clinical-Trials.gov (ISRCTN13287728; Clinical-Trials.gov: NCT02369107). This trial protocol is attached as Additional file 1.

Participants were eligible for inclusion if they were diagnosed with lung cancer, breast cancer, or gynecological cancer and scheduled to receive cisplatin, anthracycline, or taxane-based chemotherapy regimens. Patients were required to be aged 18 years or older and aged 75 years or younger. The Eastern Cooperative Oncology Group performance status was $0-2$. The expected lifetime was longer than 6 months. On the other hand, patients were excluded from joining this trial under the following conditions: severe cardiac arrhythmia, serious hepatorenal abnormal function, immune system, or hematopoietic system diseases, pregnant or lactating women. Patients with intractable vomiting caused by 
malignant brain metastases, intracranial hypertension, digestive tract obstruction, severe liver or renal dysfunction, brain tumors, cerebrovascular diseases, or other reasons couldn't take part in the research. Acupuncture is a minimally invasive therapy. Thus, patients diagnosed with coagulopathy, thrombocytopenia, or other bleeding disorders were not allowed to join. It was not suitable in our study in patients with lymphedema in selected treating areas, fear of acupuncture, or allergy to needles. Other exclusion standards included that diagnosis with depression, anxiety disorders, psychosis, sepsis, or bacteremia.

Randomization was done using permuted block randomization with a secure computer system. Randomly allocated information was sealed in opaque envelopes and delivered to each center. Random allocation was performed after eligible participants who consented in written form and complete baseline assessments. Statisticians generated the allocation sequence. Primary investigators who were responsible for enrollment, and acupuncturist who conducted treatment were aware of the allocation. Patients were not informed of the group allocation. Investigators in charge of participant interviews and stuff for statistical analysis were also blind to the assignment. Blinding will be applied to the outcome assessment.

\section{Study interventions}

All participants received chemotherapy regimens containing cisplatin, anthracycline, or taxane. Chemotherapy regimens were applied in accordance with the NCCN guidelines [17]. All patients were given pre-medications, including dexamethasone $20 \mathrm{mg}$ and ondansetron (Ondansetron Hydrochloride Injection, Ningbo Team Pharm Co. Ltd, Ningbo, China) $8 \mathrm{mg}$ intravenously as a foundation antiemetic regimen.

The trial lasted for 21 days, including 5-day treatment (from the chemotherapy initiation) and 16-day follow-up periods. All the acupuncturists who participated in this trial were major in acupuncture for more than 3 years with acupuncture licenses (Chinese medicine practitioner license) and had undergone rigorous training in conducting this trial.

Patients in this trial were randomized in a 1:1 ratio into a TA or SA group receiving the following acupuncture treatment.

\section{Treatment $A$}

Manual acupuncture was done at RN12 (Zhongwan), LR13 (Zhangmen, bilaterally), RN6 (Qihai), ST25 (Tianshu, bilaterally), PC6 (Neiguan, bilaterally), ST36 (Zusanli, bilaterally) (in Additional file 2: Table S1). All these acupuncture points excepting ST36 were stimulated manually every $10 \mathrm{~min}$. A battery-operated SDZ-II
Electronic Acupuncture Treatment Instrument (Hua Tuo medical instruments Co., Ltd. Suzhou Jiangsu, China) was connected to the needle inserted in ST36 and a skin electrode $5 \mathrm{~cm}$ from ST36 lateral on both sides. Electrical stimulation continued for $30 \mathrm{~min}$ at alternating frequencies of $2 / 100 \mathrm{~Hz}$ within the max-tolerance intensity of each patient.

\section{Treatment B}

Sham acupoints were selected for patients in this control group. To maintaining the same quantity of stimulus uniform in two groups, patients in the control group use the same kind, size, and number of needles for the control group as for the TA group. The stimulation points do not belong to traditional Chinese medicine meridians.

Participants who received TA or SA treatment twice on the day of chemotherapy initiation, and once on the consecutive 4 days. Each acupuncture session took approximately $30 \mathrm{~min}$. There are ten needles in each session for both groups. Sterile needles for single-use (Ande, made in Guizhou, China) are used in this study. 25 -gauge ( $0.25 \mathrm{~mm}$ in diameter), $40 \mathrm{~mm}$-long needles are used for both groups at limb and abdomen. Needles in the TA group will be inserted $10-35 \mathrm{~mm}$ in depth and manually manipulated to produce the peculiar sensation known as De Qi, adding electro-acupuncture stimulation. During the TA session, acupuncturists will add manipulation to the needles every other $10 \mathrm{~min}$. While in the control group, needles will be inserted about 1-2 mm indepth with no manipulation. Participants in both groups will receive ondansetron intravenously as a foundation antiemetic regimen.

The sham acupoints used in the control group.

The stimulation positions do not belong to traditional Chinese medicine meridians. The sham acupuncture points are chosen from three different areas on the body (the abdomen, the Inside of the upper limb, the lateral lower leg), which do not correspond to recognized acupuncture points and are deemed to have no therapeutic value.

Additional doses of ondansetron $4 \mathrm{mg}$ would be given if patients experienced severe nausea or vomiting.

\section{Outcome measures \\ Primary outcome measure}

The primary outcome was evaluated by The Common Terminology Criteria for Adverse Events (CTCAE 4.0), established by the US National Cancer Institute (NCI), which is a set of criteria for the standardized classification of adverse effects of drugs used in cancer therapy. It includes standards to assess nausea and vomiting. It was used at baseline, day 1 , day 2 , day 3 , day $7( \pm 1)$, day 10 $( \pm 1)$, day $14( \pm 1)$ and day $21( \pm 1)$ of the chemotherapy 
period. Patients would fill in questionnaires to record the occurring frequency and extent of CINV during the treatment period. Vomiting was defined as a projection of gastric contents and an attempt to vomit without gastric contents out from the mouth. Nausea was established as an unpleasant feeling associated with the urge to vomit. Complete control of nausea or vomiting was defined as no nausea or vomiting.

\section{Secondary outcome measure}

The secondary outcomes include the following contents. All patients were evaluated by The Eastern Cooperative Oncology Group (ECOG) [18] score, and the scores should range from 0 to 2. Hospital Anxiety and Depression scale (HADS) [19] was used to estimate the degree of anxiety and depression of patients. The HADS includes fourteen items, seven of which related to anxiety and seven related to depression. Simplified Nutritional Appetite Questionnaire (SNAQ) was applied for selfassessment of nutritional conditions [20].

ECOG and SNAQ were assessed at baseline, day 3, day $7( \pm 1)$, day $14( \pm 1)$, day $21( \pm 1)$ of the observation period. HADS were evaluated at baseline, day 3 , and day $7( \pm 1)$.

\section{Statistical analysis}

Sample size calculations were based on pilot research, in which the complete response rate of nausea and vomiting was $32 \%$ and $59 \%$ [21], respectively. The true acupuncture group was anticipated to raise the complete response rate by $25 \%$. To detect a significant difference between these two groups with a power of $80 \%(\alpha=0.05$, two-sided), factoring in a $20 \%$ withdrawal rate, the total sample size required was 134 participants with a 1:1 ratio in two groups. Randomization sequence for allocation was generated by SAS version 9.1.3 software (SAS Institute Inc., Cary, NC, USA).

Statistical analysis was performed with SPSS22.0. The baseline characteristics of the two groups were analyzed by t-tests. The categorical data such as the complete response rate of nausea/vomiting, adverse events were analyzed with Mcnemar Chi square test. If the average intensity of nausea and vomiting, the score of HADS and SNAQ followed a normal distribution, an independent $t$ test would be used. Otherwise, the data would be analyzed with the Wilcoxon rank-sum test. A P-value of less than 0.05 was considered as statistically significant.

\section{Results}

Between March 2015 to July 2017, 158 eligible patients were enrolled from Beijing Shijitan Hospital, Beijing Friendship Hospital, Beijing Hospital of Traditional Chinese Medicine, and Guang' Anmen Hospital. Guang'
Anmen Hospital was added as the fourth research center because the allocation progress was slower than we estimated.

In the 158 patients, 15 patients failed to meet the inclusion criteria, while seven declined to participate in this research. There were 136 patients joined in the study, while two patients withdrawn their signed informed consent before the assignment due to the changing of minds. Finally, 134 participants were randomly assigned into the true acupuncture group $(n=68)$ and the sham acupuncture group $(n=66)$. In the follow-up phase, nine patients (6.6\%) withdrew from this trial (3 in the true acupuncture group and 6 in the sham acupuncture group). As for the reason of drop-out, six patients transferred to another hospital for a therapeutic reason, and three patients revoked the consent forms for personal willingness. In the process of data cleaning, 5 cases were eliminated for failing to meet the inclusion criteria (over the up-age limit). Finally, 120 patients (including 62 in the true acupuncture group and 58 in the sham acupuncture group) were included in data analysis (Fig. 1). Patient baseline characteristics are shown in Table 1.

\section{Primary outcomes}

A comparison of the CINV degree in complete response rate (CRR) between two groups is exhibited in Table 2a, b). Similar emetic CRR in the 21-day observation. Although since the day 3 the CRR for nausea and vomiting in the true acupuncture group was slightly higher than that of sham acupuncture group, there is no statistical difference between two groups excepting for the CRR of nausea on the last day of chemotherapy circle $(83.9 \%$ to $67.2 \%$, respectively, $P=0.033$ ).

We evaluated the variation of nausea and vomiting degrees with CTCAE4.0 in both groups (Table 2c). Original nausea and vomiting degrees between groups showed no significant statistical difference. The nausea degree in the true acupuncture group increased in the first 2 days and reached a peak on day 2 by 0.355 points compared to baseline, and then decreased gradually till the end of the chemotherapy circle. There was a similar tendency in the nausea variation of the sham acupuncture group except that the value keeps increasing to day 3 by 0.621 points. The difference between the two groups had statistical significance since day $3(0.306$ vs. $0.621, P=0.021)$ until the end of observation (Fig. 2). The rise in the true acupuncture group at the beginning of chemotherapy was lower than that in another group, which indicating true acupuncture relieve the severity of nausea during the delay CINV and follow-up periods.

Likewise, the variation trend of vomiting in the sham acupuncture group was similar to that in nausea assessment. The vomiting degree rose by 0.448 points on day 


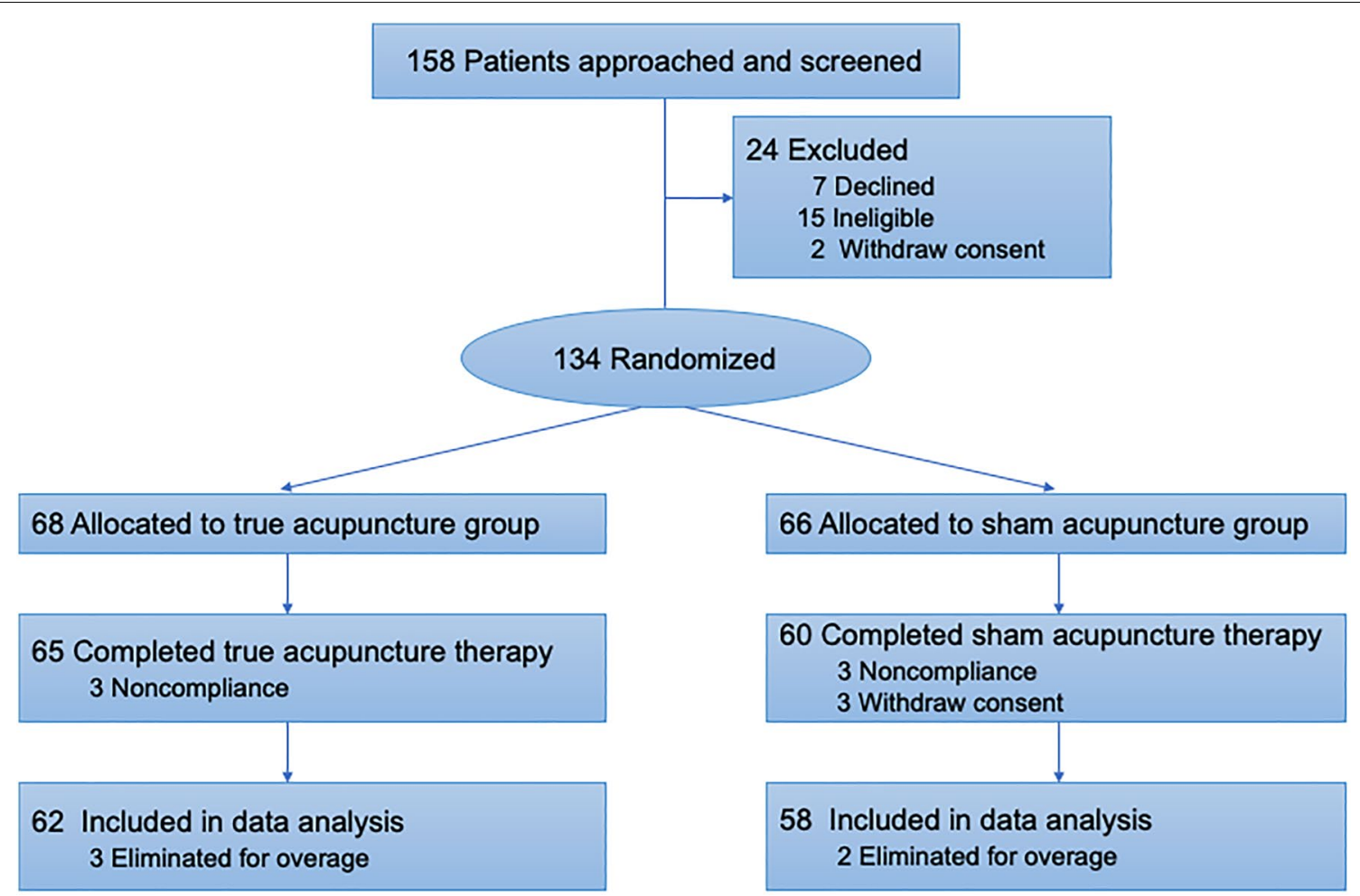

Fig. 1 Flow of Randomized Patients for the Effect of True Acuounture vs Sham Acupuncture on CINV study

2 and then went down gradually. The degree variation in the true acupuncture group continually decreased in the observation. And the values in the true acupuncture group were lower than those in the control group. The differences between the two groups were significant since day $7(0$ vs. $0.19, P=0.033)$. It demonstrated that there was no significant difference during the CINV period, but true acupuncture could relieve symptoms in the follow-up period.

\section{Secondary outcomes}

During the observation, the nutritional conditions of patients were evaluated with the SNAQ scale (Table 3). The score of SNAQ decreased on day 7 and slightly rose after that. The true acupuncture group had a higher score compared to the sham acupuncture group in the follow-up duration, and the difference was significant on day $14(21.82$ vs.20.12, $P=0.003)$ and day $21(22.39$ vs. 20.43, $P=0.001$ ). It depicted that patients receiving true acupuncture would have better nutritional status after chemotherapy.

While assessing the anxiety and depression of patients, no significant difference was found between two groups (Data were shown in Additional file 2: Table S2). All participants' ECOG score ranged from 0 to 2 .

\section{Safety assessments}

Safety was evaluated in 125 participants who were randomly enrolled and received at least 3 days of study intervention (Data were shown in Additional file 2: Table S3). Two participants in the true acupuncture group and one in the sham acupuncture group were not evaluable who withdrew consents without any intervention. One participant in the true acupuncture group and 5 in the sham acupuncture group discontinued the treatment due to transferring to other hospitals for a therapeutic reason. During the observation, 4 patients reported headache in the true acupuncture group. Diarrhea $(\mathrm{n}=2)$, constipation $(n=2)$, and headache $(n=4)$ were reported in the sham acupuncture group. One participant was evaluated with increased Urea nitrogen and Creatinine related to primary renal disease, while one in the sham acupuncture group with slightly ALT increased. No apparent adverse events related to acupuncture were observed. Patients who need an additional rescue for CINV were 17 (26.1\%) and $20(33.3 \%)$ in the true or sham acupuncture group, respectively $(P=0.435$, data were not shown in the table).

\section{Discussion}

We conducted a multi-center, single-blind, shamcontrolled prospective clinical research to investigate the effect of acupuncture in preventing 
Table 1 Baseline Characteristics for Patients in the True Acupuncture and Sham Acupuncture Groups

\begin{tabular}{|c|c|c|c|}
\hline & \multicolumn{2}{|l|}{ No. (\%) } & \multirow[t]{2}{*}{$P$ value } \\
\hline & True acupuncture group $(n=68)$ & Sham aupuncture group $(n=66)$ & \\
\hline Age, median $(95 \% \mathrm{Cl})$ & $60(56.84 \sim 60.48)$ & $58(54.99 \sim 60.04)$ & 0.653 \\
\hline Gender & & & 0.725 \\
\hline Male & $14(20.6)$ & $12(18.2)$ & \\
\hline Female & $54(79.4)$ & $54(81.8)$ & \\
\hline Study site & & & - \\
\hline Beijing hospital of traditional Chinese medicine & $22(16.4)$ & $22(16.4)$ & \\
\hline Guang'anmen hospital & $9(6.7)$ & $9(6.7)$ & \\
\hline Beijing friendship hospital & $19(14.2)$ & $16(11.9)$ & \\
\hline Beijing shijitan hospital & $18(13.4)$ & $19(14.2)$ & \\
\hline Tumor pathological type & & & 0.830 \\
\hline Breast cancer & $37(54.4)$ & $35(53.0)$ & \\
\hline Ovarian malignant carcinoma & $8(11.8)$ & $8(12.1)$ & \\
\hline Cervical carcinoma & $1(1.5)$ & $0(0.0)$ & \\
\hline Endometrial carcinoma & $2(2.9)$ & $2(3.0)$ & \\
\hline Lung cancer & $20(29.4)$ & $21(31.8)$ & \\
\hline Cancer stage & & & 0.301 \\
\hline । & $12(17.6)$ & $17(25.8)$ & \\
\hline$\|$ & $18(26.5)$ & $16(24.2)$ & \\
\hline III & $12(17.6)$ & $11(16.7)$ & \\
\hline IV & $26(38.2)$ & $21(31.8)$ & \\
\hline Prior chemotherapy & $33(48.5)$ & $30(45.5)$ & 0.721 \\
\hline \multicolumn{4}{|l|}{ Chemotherapy regimen contains } \\
\hline Cis-platinum & $28(41.1)$ & $17(25.8)$ & 0.059 \\
\hline Anthracycline & $14(20.6)$ & $19(28.8)$ & 0.271 \\
\hline Taxane & $32(47.0)$ & $38(57.6)$ & 0.223 \\
\hline
\end{tabular}

Cl confidence interval

* $P$ values are based on independent-samples t-tests comparing differences between groups in age and whether or not before chemotherapy, $P$ values in the study site, pathological tumor type, cancer stage, and chemotherapy regimen are based on Wilcoxon signed-rank test, and other $P$ values are based on $\chi^{2}$ tests

chemotherapy-induced nausea and vomiting. There is no noticeable improvement of true acupuncture in the complete response rate of CINV compared to the shamcontrol. However, TA indeed remarkedly alleviated the severities of nausea (day-3 to day-21) and emesis (day-7 to day-21) induced by chemotherapy. TA also contributed to nutritional status recovery since the day-14 after chemotherapy initiation.

Nowadays, CINV is less of a challenge for patients receiving chemotherapy owing to the development of antiemetic medications. We still need to pay special attention to the CINV occurred unreported by the data. The limitations of traditional antiemetic trials conclude evaluating outcomes only after the first circle of chemotherapy and mainly focusing on the cisplatin-based chemotherapy regimen. These designs are critical for the preciseness of researches, but also confine the application of the conclusions. Patients undergoing other drugs or multi-day chemotherapy regimens, or receiving multicycle treatments remain faced with a higher risk of CINV. Also, CINV is affected by several risk factors, such as a history of nausea/vomiting, female sex, the expectancy of CINV, younger age, anxiety, history of morning sickness, and low alcohol intake [22]. Antiemetic prophylaxis based on a risk model guide led to improved control effect of acute and delayed CINV [23]. Nevertheless, these factors are not much considered in clinical application. Compared to emesis, nausea is a more distressing symptom and less controlled [8]. More emphasis should be put on alleviating nausea to improve the QOL of cancer patients. It will promote the psychological and physical wellness of patients. Adjunctive measures require better management of CINV.

In our study, we didn't find superior CRR in the TA group but observed that TA reduced the severities of CINV and augmented the score of nutritional status. The nausea degree increased by 0.306 in the TA group while 0.621 in the SA group $(P=0.021)$ on day 
Table 2 Observed and Fitted Group Assessment of CINV in Each Group

\section{a. CTCAE assessment of CINV}

Mean (SD)

Sham acupuncture group $(n=58)$

$P$ value

True acupuncture group $(n=62)$

\section{Nausea}

Baseline

Day 1

$0.56(0.692)$

$0.40(0.528)$

0.244

0.82 (0.779)

$0.67(0.636)$

0.326

Day 2

$0.92(0.685)$

0.91 (0.779)

0.921

Day 3

$0.87(0.735)$

$1.02(0.761)$

0.288

Day $7( \pm 1)$

$0.60(0.689)$

$0.71(0.622)$

0.257

Day $10( \pm 1)$

0.37 (0.579)

$0.48(0.655)$

0.355

$0.31(0.531)$

0.40 (0.591)

0.388

Day $21( \pm 1)$

$0.18(0.426)$

$0.39(0.620)$

$0.041^{*}$

Vomiting

$0.29(0.555)$

$0.16(0.451)$

0.096

$0.52(0.695)$

$0.30(0.533)$

0.078

$0.50(0.741)$

$0.60(0.771)$

0.403

Day 2

$0.50(0.647)$

$0.57(0.704)$

0.656

Day $7( \pm 1)$

$0.29(0.584)$

$0.34(0.579)$

0.394

Day $10( \pm 1)$

0.19 (0.438)

$0.36(0.693)$

0.177

Day $14( \pm 1)$

$0.21(0.484)$

$0.31(0.681)$

0.499

$0.10(0.349)$

$0.23(0.598)$

0.183

\section{b. Completed control rate of CINV}

\section{No. (\%)}

Sham acupuncture group $(n=58)$

$P$ value

True acupuncture group $(n=62)$

\section{Nausea}

Baseline

$34(54.8)$

Day 1

$25(40.3)$

Day 2

$17(27.4)$

Day 3

$21(33.9)$

Day $7( \pm 1)$

32 (51.6)

Day $10( \pm 1)$

$42(67.7)$

Day $14( \pm 1)$

45 (72.6)

Day 21 ( \pm 1)

$52(83.9)$

$36(62.1)$

0.422

Vomiting

Baseline

47 (75.8)

$37(59.7)$

39 (62.9)

Day 2

$35(56.5)$

Day 3

$48(77.4)$

$51(82.3)$

$51(82.3)$

24 (41.4)

0.906

$20(34.5)$

0.402

16 (27.6)

0.456

$22(37.9)$

0.132

35 (60.3)

0.398

$38(65.5)$

0.402

$39(67.2)$

$0.033^{*}$

Day $7( \pm 1)$

Day $10( \pm 1)$

57 (91.9)

51 (87.9)

0.086

$42(72.4)$

0.142

$32(55.2)$

0.389

$32(55.2)$

0.888

40 (69.0)

0.295

$42(72.4)$

0.197

45 (77.6)

0.523

Day $21( \pm 1)$

$48(82.8)$

0.214

c. Variation of CINV degree evaluated by CTCAE in each group

Mean (SD)

Sham acupuncture group $(n=58)$

$P$ value

True acupuncture group $(n=62)$

\section{Nausea}

$\begin{array}{ll}\text { Day } 1 & 0.258(0.599) \\ \text { Day } 2 & 0.355(0.546) \\ \text { Day } 3 & 0.306(0.616)\end{array}$


Table 2 (continued)

c. Variation of CINV degree evaluated by CTCAE in each group

\begin{tabular}{|c|c|c|c|}
\hline & $\begin{array}{l}\text { Mean (SD) } \\
\text { True acupuncture group }(n=62)\end{array}$ & Sham acupuncture group $(n=58)$ & $P$ value \\
\hline Day 7 ( \pm 1$)$ & $0.032(0.724)$ & $0.310(0.654)$ & $0.030^{*}$ \\
\hline Day $10( \pm 1)$ & $-0.194(0.649)$ & $0.086(0.539)$ & $0.011^{*}$ \\
\hline Day $14( \pm 1)$ & $-0.258(0.676)$ & $0.000(0.375)$ & $0.011^{*}$ \\
\hline Day 21 ( \pm 1) & $-0.387(0.662)$ & $-0.017(0.477)$ & $0.001^{*}$ \\
\hline \multicolumn{4}{|l|}{ Vomiting } \\
\hline Day 1 & $0.226(0.556)$ & $0.138(0.348)$ & 0.298 \\
\hline Day 2 & $0.210(0.631)$ & $0.448(0.776)$ & 0.068 \\
\hline Day 3 & $0.210(0.604)$ & $0.414(0.676)$ & 0.085 \\
\hline Day 7 ( \pm 1$)$ & $0.000(0.444)$ & $0.190(0.512)$ & $0.033^{*}$ \\
\hline Day $10( \pm 1)$ & $-0.097(0.503)$ & $0.207(0.585)$ & $0.003^{*}$ \\
\hline Day $14( \pm 1)$ & $-0.081(0.552)$ & $0.155(0.556)$ & $0.021^{*}$ \\
\hline Day 21 ( \pm 1) & $-0.194(0.507)$ & $0.069(0.454)$ & $0.003^{*}$ \\
\hline
\end{tabular}

CINV Chemotherapy Induced Nausea and Vomiting, CTCAE the Common Terminology Criteria for Adverse Events, SD standard deviation

${ }^{*} P$ values are based on independent-samples $t$ tests comparing differences in between-group means

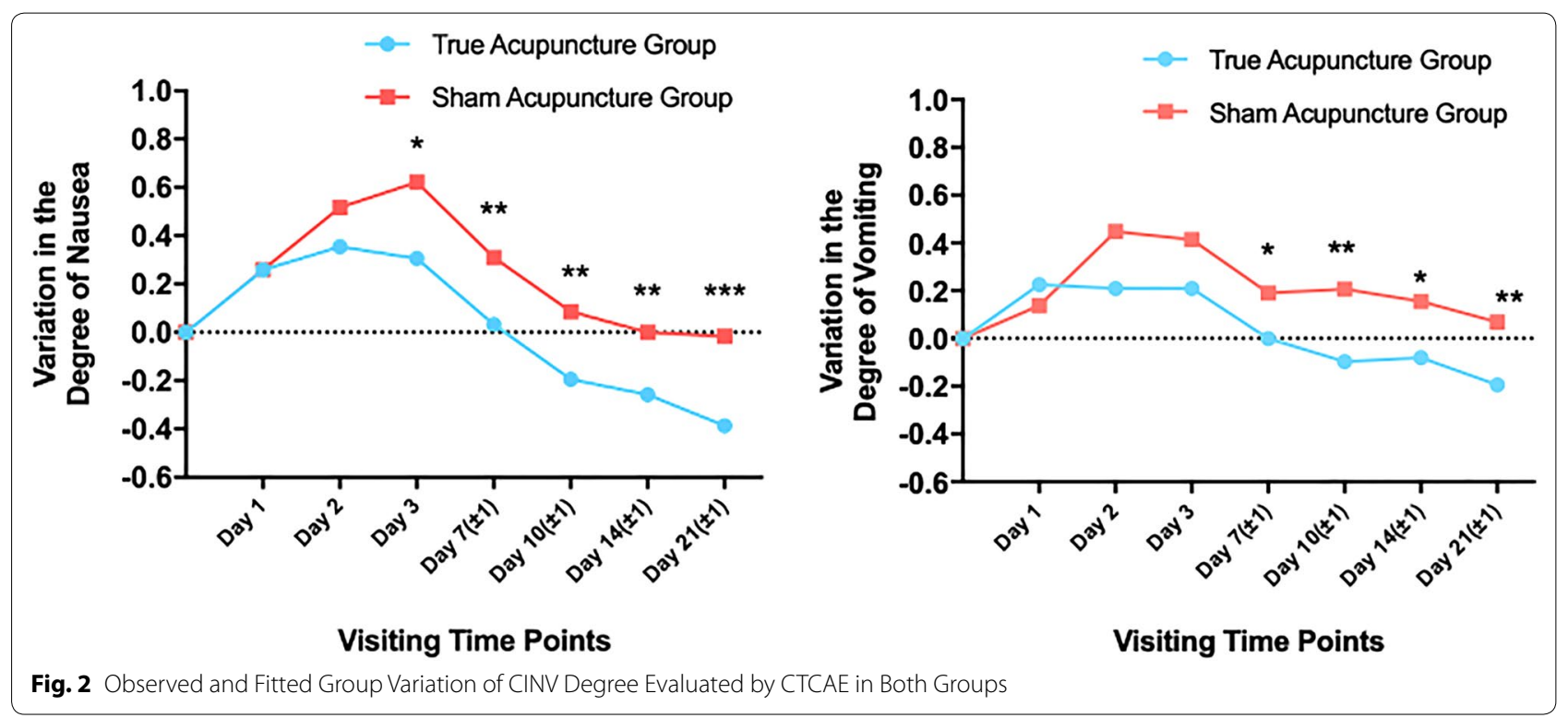

Table 3 Observed and Fitted Group Results for Simplified Nutritional Appetite Questionnaire assessment

\begin{tabular}{|c|c|c|c|c|c|}
\hline & True acupunc & $n=62)$ & Sham Acupun & $(n=58)$ & $P$-value \\
\hline & Mean (SD) & $95 \% \mathrm{Cl}$ & Mean (SD) & $95 \% \mathrm{Cl}$ & \\
\hline Day 1 & $20.145(3.463)$ & $19.27 \sim 21.02$ & $21.138(2.453)$ & $20.49 \sim 21.78$ & 0.071 \\
\hline Day $7( \pm 1)$ & $19.113(3.052)$ & $18.34 \sim 19.89$ & $18.983(3.301)$ & $18.11 \sim 19.85$ & 0.823 \\
\hline Day $10( \pm 1)$ & $20.806(3.274)$ & $19.98 \sim 21.64$ & $19.897(2.942)$ & $19.12 \sim 20.67$ & 0.113 \\
\hline Day $14( \pm 1)$ & $21.823(3.262)$ & $20.99 \sim 22.65$ & $20.121(2.728)$ & $19.40 \sim 20.84$ & $0.003^{*}$ \\
\hline Day $21( \pm 1)$ & $22.387(3.138)$ & $21.59 \sim 23.18$ & $20.431(2.872)$ & $19.68 \sim 21.19$ & $0.001 *$ \\
\hline
\end{tabular}

$\mathrm{SD}$ standard deviation, $\mathrm{Cl}$ confidence interval

*P values are based on independent-samples t-tests comparing differences in between-group means 
3. Although there was no significant improvement in vomiting in the $120 \mathrm{~h}$ after chemotherapy initiation, TA reduced the vomiting degree since day 7(0 vs.0.190, $P=0.033)$. These results are consistent with findings by other researchers [24, 25]. Acupuncture didn't exhibit a promising effect in reducing the occurrence of CINV, which might due to acupuncture have less influence on preventing CINV from taking place than antiemetic drugs. The impact of acupuncture took a longer time to show up and mainly manifested in modulating gastrointestinal functions. The mechanisms related to electroacupuncture were analyzed with animal models. Electroacupuncture at Neiguan (PC 6) and Jianshi (PC 5) could reduce nausea and vomiting during chemotherapy, which was possibly associated with the deduction of 5- $\mathrm{HT}_{3}$ and dopamine [26]. It was also confirmed that electro-acupuncture(EA) at Zhongwan (CV12) alleviated the cisplatin-induced anorexia in rats by reducing the levels of plasma monoamine neurotransmitters 5-hydroxytryptamine, 5-hydroxyindoleacetic acid, dopamine, and norepinephrine; as well as upregulating the expression of ghrelin and neuropeptide $Y$ [27]. In another rat model, EA at CV12 not only attenuated the cisplatin-induced increase in 5-HT but also suppressed neuronal activation marker c-Fos expression in the nucleus tractus solitarius (NTS) [28]. Acupuncture exhibits a positive effect on alleviating nausea and promoting the nutrition intake, which compensates for the deficiency of antiemetic medication. Our results also indicated that acupuncture functioned after a period of time of treatment in general cases. It might be an effective strategy to initiate acupuncture ahead of chemotherapy for patients with a high risk of CINV. What's more, for the sake of widespread implementation, our research allocated patients with multiple types of advanced cancers receiving various regimens, multi-circle of chemotherapy. TA and SA groups were comparable with similar participant composition with each factor. However, it is not sufficient to draw a solid conclusion for which population is more suitable to receive acupuncture during chemotherapy. An enriched enrollment strategy was indicated for future studies.

To eliminate the impact of the subjective factor, patients in both groups received acupuncture treatment with the same frequency, needle-retained time, an interval of treatment to achieve effective masking. Investigators in charge of participant interviews were also blind to the assignment. Whereas, the principal investigator who was responsible for distributing tasks and acupuncturists were not blinded. We also applied blind evaluation in statistical analysis. All these measures contributed to addressing reliable conclusions.

\section{Strengths and Limitations}

This study has several strengths. The intervention was conducted by several acupuncturists undergoing rigorous standardized training to make sure the operational consistency in this trial. Patients were treated at multiple sites with different stages of cancer, different cycles of chemotherapy, which more like the situations in the real world and increased the generalizability of the findings. The observation not only for the CINV phase (5 days since chemotherapy administration) but also including the follow-up 16 days. It made it possible to evaluate the effect of acupuncture in the whole chemotherapy cycle.

This study also has several limitations. First, in the study design, waitlist control was in need to eliminate the influence of the physiological effect of sham acupuncture [29]. Second, some participants in the study withdrawing for different reasons might impact the evaluation of the effects of acupuncture. Third, enrollment progressed slower than expected due to the patients' turnover in the hospitals, limiting the enthusiasm of patients for participating in a randomized clinical trial. Fourth, there may exist the possibility that rare patients were aware of their allocation due to possessing a good knowledge of Chinese Medicine.

Article Summary-Strengths and limitations of this study

1. Acupuncturists and researchers received rigorous standardized training which guaranteed the operational consistency and the results reliability of the study.

2. The participants met the inclusion criteria of diversity increased the generalizability of the findings.

3. The waitlist control was in need to eliminate the influence of the physiological effect of sham acupuncture.

\section{Conclusion}

In conclusion, the current study found the effect of acupuncture in preventing CINV occurring is relatively modest. Still, it was feasible and practical to apply acupuncture for alleviating chemotherapy-induced nausea and vomiting and improving nutritional status of patients with advanced cancer.

\section{Supplementary information}

Supplementary information accompanies this paper at https://doi. org/10.1186/s13020-020-00333-x.

Additional file 1. Study protocol.

Additional file 2. Acupuncture therapy regimens and supplementary results. 


\section{Abbreviations}

CINV: Chemotherapy-Induced Nausea and Vomiting; CTCAE: The common terminology criteria for adverse events; ECOG: The eastern cooperative oncology group score; SNAQ: Simplified nutritional appetite questionnaire; HADS: Hospital anxiety and depression scale; HEC: Highly emetogenic chemotherapy; MEC: Moderately emetogenic chemotherapy; $\mathrm{NCl}$ : National cancer institute; ZPS: Zubrod score; AEs: Adverse events; SPSS: Statistical package of social sciences software; QOL: Quality of life; $5-\mathrm{HT}_{3}$ : 5-Hydroxytryptamine type 3; RAs: Receptor antagonists; NTS: The nucleus tractus solitarius; NK ${ }_{1}$ : Neurokinin-1.

\section{Acknowledgements}

We thank Beijing Hospital of Traditional Chinese Medicine, Beijing Friendship Hospital, Beijing Shijitan Hospital, and Guang'Anmen Hospital, all clinicians who consented or referred their patients to our study, all acupuncturists who conducted intervention conscientiously and rigorously, and all researchers who performed the study responsibly. Most importantly, we thank all the participants for their dedication and support to this study.

\section{Protocol}

The protocol of this research is available online: (https://trialsjournal.biome dcentral.com/articles/10.1186/s13063-017-1927-2).

\section{Authors' contributions}

QWL drafted the manuscript and implemented the research plan. MWY and XMW conceived and designed this trial. GWY provided a critical contribution to the organization and cooperation of this multi-center task. NX, WRX, YZ, PYC, LY, QF, ZY participated in the enrollment of the patients. QWL, HW, CXZ recruited the patients and collected data. All authors read and approved the final manuscript.

\section{Funding}

This work is financed by grants from the Beijing Municipal Administration of Hospitals (No. XM201410).

\section{Data sharing statement}

All relevant data are within the paper and its supporting information files.

\section{Ethical approval and consent to participate}

The protocol was carried out according to GCP guidelines and in accordance with the principles of the Declaration of Helsinki. This study had been approved by the Research Ethical Committee of Beijing Hospital of Traditional Chinese Medicine Affiliated to Capital Medical University on 19 November 2014 (ref: 2014BL-067). All participants had signed written informed consent.

\section{Consent for publication}

Not applicable.

\section{Competing interests}

The authors declare that they have no competing interests.

\section{Author details}

${ }_{1}^{1}$ Beijing Hospital of Traditional Chinese Medicine Affiliated with Capital Medical University, No 23, Back Road of Art Gallery, Dong Cheng District, Beijing 100010, China. ${ }^{2}$ Beijing University of Chinese Medicine, No. 11, North 3rd Ring East Road, Chaoyang District, Beijing 100029, China.

Received: 31 January 2020 Accepted: 14 May 2020

Published online: 03 June 2020

\section{References}

1. Navari RM, Aapro M. Antiemetic prophylaxis for chemotherapy-induced nausea and vomiting. New Engl J Med. 2016;374(14):1356-67.

2. Hesketh PJ, Kris MG, Basch E, Bohlke K, Barbour SY, Clark-Snow RA, et al. Antiemetics: American society of clinical oncology clinical practice guideline update. J Clin Oncol. 2017;35(28):3240-61.

3. Hashimoto $\mathrm{H}$, Abe M, Tokuyama O, Mizutani H, Uchitomi Y, Yamaguchi $\mathrm{T}$, et al. Olanzapine $5 \mathrm{mg}$ plus standard antiemetic therapy for the prevention of chemotherapy-induced nausea and vomiting (J-FORCE): a multicentre, randomized, double-blind, placebo-controlled, phase 3 trial. Lancet Oncol. 2020;21(2):242-9.

4. Roscoe JA, Heckler CE, Morrow GR, Mohile SG, Dakhil SR, Wade JL, et al. Prevention of delayed nausea: a University of Rochester Cancer Center community clinical oncology program study of patients receiving chemotherapy. J Clin Oncol. 2012;30(27):3389-95.

5. Clark-Snow R, Affronti ML, Rittenberg CN. Chemotherapy-induced nausea and vomiting (CINV) and adherence to antiemetic guidelines: results of a survey of oncology nurses. Support Care Cancer. 2018;26(2):557-64.

6. Grunberg SM. Obstacles to the implementation of antiemetic guidelines. J Natl Compr Cancer Net. 2009;7(5):601-5.

7. Salsman JM, Grunberg SM, Beaumont JL, Rogers M, Paul D, Clayman ML, et al. Communicating about chemotherapy-induced nausea and vomiting: a comparison of patient and provider perspectives. J Nat Compr Cancer Net. 2012;10(2):149-57.

8. Ng TL, Hutton B, Clemons M. Chemotherapy-induced nausea and vomiting: time for more emphasis on nausea? Oncologist. 2015;20(6):576-83.

9. Navari RM, Schwartzberg LS. Evolving role of neurokinin 1-receptor antagonists for chemotherapy-induced nausea and vomiting. Onco Targets Ther. 2018;11:6459-78.

10. Lyman GH, Greenlee H, Bohlke K, Bao T, DeMichele AM, Deng GE, et al. Integrative therapies during and after breast cancer treatment: ASCO endorsement of the SIO clinical practice guideline. J Clin Oncol. 2018;36(25):2647-55.

11. Magnusson M, Hoglund P, Johansson K, Jonsson C, Killander F, Malmstrom $P$, et al. Pentoxifylline and vitamin $E$ treatment for prevention of radiation-induced side-effects in women with breast cancer: a phase two, double-blind, placebo-controlled randomised clinical trial (Ptx-5). Eur J Cancer. 2009;45(14):2488-95.

12. Rithirangsriroj K, Manchana T, Akkayagorn L. Efficacy of acupuncture in prevention of delayed chemotherapy induced nausea and vomiting in gynecologic cancer patients. Gynecol Oncol. 2015;136(1):82-6.

13. Shen J, Wenger N, Glaspy J, Hays RD, Albert PS, Choi C, et al. Electroacupuncture for control of myeloablative chemotherapy-induced emesis: a randomized controlled trial. JAMA. 2000;284(21):2755-61.

14. Garcia MK, McQuade J, Haddad R, Patel S, Lee R, Yang P, et al. Systematic review of acupuncture in cancer care: a synthesis of the evidence. J Clin Oncol. 2013;31(7):952-60.

15. Murphy JF. Consort 2010 statement on randomised controlled trials. Ir Med J. 2010;103(5):132.

16. MacPherson H, Altman DG, Hammerschlag R, Youping L, Taixiang W, White $A$, et al. Revised standards for reporting interventions in clinical trials of acupuncture (STRICTA): extending the CONSORT statement. J Evid Based Med. 2010;3(3):140-55.

17. Ettinger DS, Bierman PJ, Bradbury B, Comish CC, Ellis G, Ignoffo RJ, et al. Antiemesis. J Nat Compr Cancer Net. 2007;5(1):12-33.

18. Oken MM, Creech RH, Tormey DC, Horton J, Davis TE, McFadden ET, et al. Toxicity and response criteria of the eastern cooperative oncology group. Am J Clin Oncol. 1982;5(6):649-55.

19. Zigmond AS, Snaith RP. The hospital anxiety and depression scale. Acta Psychiatr Scand. 1983;67(6):361-70.

20. Wilson MM, Thomas DR, Rubenstein LZ, Chibnall JT, Anderson S, Baxi A, et al. Appetite assessment: simple appetite questionnaire predicts weight loss in community-dwelling adults and nursing home residents. Am J Clin Nutr. 2005;82(5):1074-81.

21. Yang $X$, Zhang S. A randomized control study on the clinical effects of ramosetron in prophylaxis of nausea and vomiting induced by cisplatin chemotherapy in patients with lung cancer. Zhongguo fei ai za zhi. 2005;8(4):322-5.

22. Mosa ASM, Hossain AM, Lavoie BJ, Yoo I. Patient-related risk factors for chemotherapy-induced nausea and vomiting: a systematic review. Front Pharm. 2020;11:329.

23. Clemons M, Bouganim N, Smith S, Mazzarello S, Vandermeer L, Segal R, et al. Risk model-guided antiemetic prophylaxis vs physician's choice in patients receiving chemotherapy for early-stage breast cancer: a randomized clinical trial. JAMA Oncol. 2016;2(2):225-31.

24. Xie J, Chen LH, Ning ZY, Zhang CY, Chen H, Chen Z, et al. Effect of transcutaneous electrical acupoint stimulation combined with palonosetron on chemotherapy-induced nausea and vomiting: a single-blind, randomized, controlled trial. Chin J Cancer. 2017;36(1):6. 
25. Shen Y, Liu L, Chiang JS, Meng Z, Garcia MK, Chen Z, et al. Randomized, placebo-controlled trial of $\mathrm{K} 1$ acupoint acustimulation to prevent cisplatin-induced or oxaliplatin-induced nausea. Cancer. 2015;121(1):84-92.

26. Zhang X, Fan YH. Effects of electroacupuncture on chemotherapyinduced nausea and vomiting and its mechanism. Zhongguo zhen jiu. 2014;34(11):1061-4.

27. Baek JY, Trinh TA, Huh W, Song JH, Kim HY, Lim J, et al. Electro-acupuncture alleviates cisplatin-induced anorexia in rats by modulating ghrelin and monoamine neurotransmitters. Biomolecules. 2019;9(10):624

28. Cui Y, Wang L, Shi G, Liu L, Pei P, Guo J. Electroacupuncture alleviates cisplatin-induced nausea in rats. Acupunct Med. 2016;34(2):120-6.
29. Kong J, KaptchukTJ, Polich G, Kirsch I, Vangel M, Zyloney C, et al. Expectancy and treatment interactions: a dissociation between acupuncture analgesia and expectancy evoked placebo analgesia. Neurolmage. 2009;45(3):940-9.

\section{Publisher's Note}

Springer Nature remains neutral with regard to jurisdictional claims in published maps and institutional affiliations.
Ready to submit your research? Choose BMC and benefit from:

- fast, convenient online submission

- thorough peer review by experienced researchers in your field

- rapid publication on acceptance

- support for research data, including large and complex data types

- gold Open Access which fosters wider collaboration and increased citations

- maximum visibility for your research: over $100 \mathrm{M}$ website views per year

At BMC, research is always in progress.

Learn more biomedcentral.com/submissions 